Mathematical Modelling AND AnAlysis

Volume 18 Number 4, September 2013, 529-536

http://dx.doi.org/10.3846/13926292.2013.840867

(c) Vilnius Gediminas Technical University, 2013
Publisher: Taylor\&Francis and VGTU

http://www.tandfonline.com/TMMA

Print ISSN: 1392-6292

Online ISSN: 1648-3510

\title{
Approximate Solution to a Multi-Point Boundary Value Problem Involving Nonlocal Integral Conditions by Reproducing Kernel Method
}

\section{Kemal Özen ${ }^{a, b}$ and Kamil Oruçoğlu ${ }^{a}$}

${ }^{a}$ Department of Mathematics, İstanbul Technical University

Maslak, 34469 İstanbul, Turkey

${ }^{b}$ Department of Mathematics, Namık Kemal University

Değirmenaltı, 59030 Tekirdağ, Turkey

E-mail(corresp.): ozenke@itu.edu.tr, kozen@nku.edu.tr

E-mail: koruc@itu.edu.tr

Received January 12, 2012; revised August 7, 2013; published online September 1, 2013

\begin{abstract}
In this work, we investigate a sequence of approximations converging to the existing unique solution of a multi-point boundary value problem(BVP) given by a linear fourth-order ordinary differential equation with variable coefficients involving nonlocal integral conditions by using reproducing kernel method(RKM). Obtaining the reproducing kernel of the reproducing kernel space by using the original conditions given directly by RKM may be troublesome and may introduce computational costs. Therefore, in these cases, initially considering more admissible conditions which will allow the reproducing kernel to be computed more easily than the original ones and then taking into account the original conditions lead us to satisfactory results. This analysis is illustrated by a numerical example. The results demonstrate that the method is still quite accurate and effective for the cases with both derivative and integral conditions even if the accuracy is less compared to the cases with just derivative conditions.
\end{abstract}

Keywords: reproducing kernel space, differential equation, nonlocal integral condition, boundary value problem, nonlocal boundary condition.

AMS Subject Classification: 34B10; 34B05; 47B32; 46E22.

\section{Introduction}

Nonlocal multi-point boundary value problems arise in applied mathematics, physics, engineering and the various areas of mechanics such as theory of elasticity, theory of elastic stability and theory of plates and shells [14]. The mathematical models for the vibrations of wires and bridges composed of many parts of different densities involve such problems. 
Finding solutions analytically to multi-point boundary value problems represented by linear ordinary differential equations with variable coefficients involving nonlocal boundary conditions can be based on determining of some fundamental solutions such as Green's function or Green's functional [10,11, $12,13,15]$. On the other hand, the methods yielding effective approximate solutions have started to become important and valuable with the development of computation tools in recent years. One of these methods is RKM which is based on reproducing kernel theory and Fourier method. The difference between RKM and Fourier method emanates from the way different systems of complete functions are generated for the solution of the problem by each method.

Reproducing kernel theory has many potential applications in numerical analysis of differential equations $[1,2,3,4,5,6,7,9,17]$. In this context, the presented method in [16] for solving the problem represented by an ordinary differential equation with only derivative conditions is implemented to obtain the approximate solution to linear fourth-order multi-point BVP governed by an ordinary differential equation with both derivative and integral conditions in this work.

The problem for which the coefficients and right-hand side expressions are chosen such that its unique solution will exist is as follows:

$$
\begin{gathered}
u^{(4)}(x)+\sum_{i=0}^{3} A_{i}(x) u^{(i)}(x)=f(x), \quad 0 \leq x \leq 1, \\
V_{i} u \equiv \sum_{j=0}^{3} \alpha_{i j} u^{(j)}\left(\beta_{j}\right)+\sum_{j=0}^{3} \int_{0}^{1} \mu_{i j}(s) u^{(j)}(s) d s=z_{i} \quad(i=0,1,2,3),
\end{gathered}
$$

where $A_{i}(x) \in C^{i}[0,1], f(x) \in W_{2}^{1}[0,1], u(x) \in W_{2}^{5}[0,1]$ and $\alpha_{i j}, z_{i}, \beta_{j}$ for $i, j=0,1,2,3$ are real numbers such that $0<\beta_{0}<\beta_{1}<\beta_{2}<\beta_{3}<1$. The function $\mu_{i j}(i, j=0,1,2,3)$ is defined on $[0,1]$ such that the product $\mu_{i j} u^{(j)}$ $(i, j=0,1,2,3)$ is integrable on $[0,1]$. Here $u^{(i)}$ for $i=1,2,3,4$ denotes the derivative of order $i$ for $u$ with respect to its variable and $u^{(0)}=u$. $W_{2}^{5}[0,1]$ and $W_{2}^{1}[0,1]$ are the reproducing kernel spaces which are defined in Section 3 . As can be seen, form (1.2) for nonlocal conditions of the problem is a general form of the linear conditions for the problem with such a fourth-order linear ordinary differential equation. Many linear conditions arising in modelling of many physical phenomena by such an equation are the specific forms of (1.2).

\section{The Solution Algorithm for Problem (1.1)-(1.2)}

The following steps derived from RKM may be used to avoid the computational cost incurred from constructing the reproducing kernels of the spaces by using given original conditions directly [16].

Step 1: Construct auxiliary boundary conditions as follows:

$$
u(0)=\gamma_{0}, \quad u(1)=\gamma_{1}, \quad u^{\prime}(0)=\gamma_{2}, \quad u^{\prime}(1)=\gamma_{3},
$$

where $\gamma_{0}, \gamma_{1}, \gamma_{2}$ and $\gamma_{3}$ are constants to be determined in Step 3. 
Step 2: Solve the following two-point BVP by RKM:

$$
\begin{gathered}
u^{(4)}(x)+\sum_{i=0}^{3} A_{i}(x) u^{(i)}(x)=f(x), \quad 0 \leq x \leq 1, \\
u(0)=\gamma_{0}, \quad u(1)=\gamma_{1}, \quad u^{\prime}(0)=\gamma_{2}, \quad u^{\prime}(1)=\gamma_{3} .
\end{gathered}
$$

In order to homogenize the conditions, a new unknown function $v(x)=$ $u(x)-\phi(x)$ where $\phi(x)$ is a third-order polynomial satisfying $\phi(0)=\gamma_{0}$, $\phi(1)=\gamma_{1}, \phi^{\prime}(0)=\gamma_{2}$ and $\phi^{\prime}(1)=\gamma_{3}$ is considered. Thus,

$$
\phi(x)=(-1+x)^{2}(1+2 x) \gamma_{0}+x\left((3-2 x) x \gamma_{1}+(-1+x)\left((-1+x) \gamma_{2}+x \gamma_{3}\right)\right) .
$$

The next aim is to solve the reduced problem of obtaining a function $v(x)$ satisfying

$$
\begin{gathered}
v^{(4)}(x)+\sum_{i=0}^{3} A_{i}(x) v^{(i)}(x)=g\left(x, \gamma_{0}, \gamma_{1}, \gamma_{2}, \gamma_{3}\right), \quad 0 \leq x \leq 1, \\
v(0)=0, \quad v(1)=0, \quad v^{\prime}(0)=0, \quad v^{\prime}(1)=0
\end{gathered}
$$

where

$$
g\left(x, \gamma_{0}, \gamma_{1}, \gamma_{2}, \gamma_{3}\right)=f(x)-\sum_{i=0}^{3} A_{i}(x) \phi^{(i)}(x) .
$$

By using the RKM $[1,3]$, the solution and the $n$-approximation to the problem (2.5)-(2.6) can be obtained respectively:

$$
\begin{aligned}
v(x) & =\sum_{i=1}^{\infty} \sum_{k=1}^{i} \beta_{i k} g\left(x_{k}, \gamma_{0}, \gamma_{1}, \gamma_{2}, \gamma_{3}\right) \overline{\psi_{i}}(x), \\
v_{n}(x) & =\sum_{i=1}^{n} \sum_{k=1}^{i} \beta_{i k} g\left(x_{k}, \gamma_{0}, \gamma_{1}, \gamma_{2}, \gamma_{3}\right) \overline{\psi_{i}}(x),
\end{aligned}
$$

where $\beta_{i k}, x_{k}$ and $\overline{\psi_{i}}(x)$ are all given. The details can be seen in next section. Thus, the solution and the $n$-approximation to the problem $(2.2)-(2.3)$ can be obtained respectively:

$$
\begin{gathered}
u(x)=\phi(x)+\sum_{i=1}^{\infty} \sum_{k=1}^{i} \beta_{i k} g\left(x_{k}, \gamma_{0}, \gamma_{1}, \gamma_{2}, \gamma_{3}\right) \overline{\psi_{i}}(x) \\
u_{n}(x)=\phi(x)+\sum_{i=1}^{n} \sum_{k=1}^{i} \beta_{i k} g\left(x_{k}, \gamma_{0}, \gamma_{1}, \gamma_{2}, \gamma_{3}\right) \overline{\psi_{i}}(x)
\end{gathered}
$$

Step 3: When the original boundary conditions of (1.1)-(1.2) are implemented for $u_{n}(x)$ we have

$$
\sum_{j=0}^{3} \alpha_{i j} u_{n}^{(j)}\left(\beta_{j}\right)+\sum_{j=0}^{3} \int_{0}^{1} \mu_{i j}(s) u_{n}^{(j)}(s) d s=z_{i} \quad(i=0,1,2,3) .
$$


This last system is a system of four linear equations containing the unknowns $\gamma_{0}, \gamma_{1}, \gamma_{2}$ and $\gamma_{3}$. Hence, these unknowns can be determined easily.

Step 4: The unknowns are substituted into $u_{n}(x)$ in (2.8). Finally, the $n$ approximate solution to the problem (1.1)-(1.2) can be obtained.

\section{Solution to Problem (2.5)-(2.6) by the RKM}

By $[1,2,3]$, we have the following definitions:

Definition 1. Let $E$ and $C$ be respectively a nonempty set and set of complex numbers. A function $R: E \times E \rightarrow C$ is a reproducing kernel of a Hilbert space $H$ if and only if

(a) $R(\cdot, t) \in H$ for $\forall t \in E$,

(b) $\langle\varphi, R(\cdot, t)\rangle=\varphi(t)$ for $\forall t \in E$ and $\forall \varphi \in H$.

Definition 2. A Hilbert space involving a reproducing kernel is called a reproducing kernel Hilbert space or reproducing kernel space.

First, by using the RKM [3], the reproducing kernel space $W_{2}^{5}[0,1]$ is constructed such that each of its elements complies with the boundary conditions in (2.6). The space defined as $W_{2}^{5}[0,1]=\left\{u(x) \mid u(x), u^{\prime}(x), u^{\prime \prime}(x), u^{\prime \prime \prime}(x), u^{(4)}(x)\right.$ are absolutely continuous real valued functions, $u^{(5)}(x) \in L^{2}[0,1], u(0)=0$, $\left.u(1)=0, u^{\prime}(0)=0, u^{\prime}(1)=0\right\}$ is a Hilbert space [3].

The inner product and norm in $W_{2}^{5}[0,1]$ are defined respectively as follows:

$$
\begin{aligned}
& \langle u(y), v(y)\rangle_{W_{2}^{5}}=\sum_{i=0}^{2} u^{(i)}(0) v^{(i)}(0)+\sum_{i=0}^{1} u^{(i)}(1) v^{(i)}(1)+\int_{0}^{1} u^{(5)} v^{(5)} d y, \\
& \|u\|_{W_{2}^{5}}=\sqrt{\langle u, u\rangle_{W_{2}^{5}}}
\end{aligned}
$$

where $u, v \in W_{2}^{5}[0,1], u^{(0)}=u$ and $v^{(0)}=v$.

According to $[3,16]$, the reproducing kernel for $W_{2}^{5}[0,1]$ is in the following form

$$
R_{x}(y)= \begin{cases}R_{11}(x, y) & \text { for } x \geq y \\ R_{12}(x, y) & \text { for } x<y\end{cases}
$$

where $R_{11}(x, y)=\frac{1}{362880}(x-1)^{2} y^{2}\left(y(3 y-4) x^{7}+(19-12 y) y x^{6}+3 y(3 y-\right.$ 10) $x^{5}+5 y(6 y+1) x^{4}+5(8-15 y) y x^{3}+3\left(y^{7}-6 y^{6}+12 y^{5}+30240 y^{2}-60480 y+\right.$ $\left.30240) x^{2}+y^{6}(2 y-9) x+y^{7}\right)$ and $R_{12}(x, y)=R_{11}(y, x)$.

Another similarly defined reproducing kernel space which is also a Hilbert space is $W_{2}^{1}[0,1]=\{u(x) \mid u(x)$ is absolutely continuous real valued function, $\left.u^{\prime}(x) \in L^{2}[0,1]\right\}[8]$. The inner product and norm in $W_{2}^{1}[0,1]$ are defined respectively as follows:

$$
\langle u(x), v(x)\rangle_{W_{2}^{1}}=\int_{0}^{1}\left(u(x) v(x)+u^{\prime}(x) v^{\prime}(x)\right) d x, \quad\|u\|_{W_{2}^{1}}=\sqrt{\langle u, u\rangle_{W_{2}^{1}}},
$$


where $u(x), v(x) \in W_{2}^{1}[0,1]$. Its reproducing kernel [8] is

$$
\bar{R}_{x}(y)=\frac{1}{2 \sinh (1)}[\cosh (x+y-1)+\cosh (|x-y|-1)] .
$$

If $(L v)(x) \equiv v^{(4)}(x)+\sum_{i=0}^{3} A_{i}(x) v^{(i)}(x)$ in $(2.5)$, then $L: W_{2}^{5}[0,1] \rightarrow$ $W_{2}^{1}[0,1]$ is a bounded linear operator $[3] . \varphi_{i}(x)=\bar{R}_{x_{i}}(x)$ and $\psi_{i}(x)=L^{*} \varphi_{i}(x)$ where $L^{*}$ denotes the adjoint operator of $L$. The orthonormal system $\left\{\bar{\psi}_{i}(x)\right\}_{i=1}^{\infty}$ in $W_{2}^{5}[0,1]$ can be obtained from the system $\left\{\psi_{i}(x)\right\}_{i=1}^{\infty}$ in $W_{2}^{5}[0,1]$ by using Gram-Schmidt orthonormalization method:

$$
\bar{\psi}_{i}(x)=\sum_{k=1}^{i} \beta_{i k} \psi_{k}(x) \quad\left(\beta_{i i}>0, i=1,2, \ldots\right),
$$

where $\beta_{i k}$ are the orthogonalization coefficients.

According to $[1,2,3]$, we have the following theorems:

Theorem 1. For $(2.5)-(2.6)$, if $\left\{x_{i}\right\}_{i=1}^{\infty}$ is dense on $[0,1]$, then $\left\{\psi_{i}(x)\right\}_{i=1}^{\infty}$ is a complete system in $W_{2}^{5}[0,1]$ and $\psi_{i}(x)=\left.L_{y} R_{x}(y)\right|_{y=x_{i}}$ where the operator $L_{y}$ denotes that the operator $L$ applies to the function of $y$.

It can be deduced that $\psi_{i}(x)=\left(L^{*} \varphi_{i}\right)(x)=\left\langle\left(L^{*} \varphi_{i}\right)(y), R_{x}(y)\right\rangle_{W_{2}^{5}}=\left\langle\varphi_{i}(y)\right.$, $\left.L_{y} R_{x}(y)\right\rangle_{W_{2}^{1}}=\left\langle\bar{R}_{x_{i}}(y), L_{y} R_{x}(y)\right\rangle_{W_{2}^{1}}=\left.L_{y} R_{x}(y)\right|_{y=x_{i}}$ and $\psi_{i}(x) \in W_{2}^{5}[0,1]$ easily. If $\left\langle v(x), \psi_{i}(x)\right\rangle_{W_{2}^{5}}=0(i=1,2, \ldots)$ for each fixed $v(x) \in W_{2}^{5}[0,1]$, then

$$
\begin{aligned}
\left\langle v(x), \psi_{i}(x)\right\rangle_{W_{2}^{5}} & =\left\langle v(x),\left(L^{*} \varphi_{i}\right)(x)\right\rangle_{W_{2}^{5}} \\
& =\left\langle L v(\cdot), \varphi_{i}(\cdot)\right\rangle_{W_{2}^{1}}=\left\langle L v(\cdot), \bar{R}_{x_{i}}(\cdot)\right\rangle_{W_{2}^{1}} \\
& =(L v)\left(x_{i}\right)=0 .
\end{aligned}
$$

Theorem 2. If $\left\{x_{i}\right\}_{i=1}^{\infty}$ is dense on $[0,1]$ and the exact solution $v(x)$ of problem (2.5)-(2.6) exists and is unique, then it can be expanded in terms of a Fourier series about orthonormal system $\left\{\bar{\psi}_{i}(x)\right\}_{i=1}^{\infty}$ as in (2.7) by noting that $\left\langle w(x), \bar{R}_{x_{i}}(x)\right\rangle_{W_{2}^{1}}=w\left(x_{i}\right)$ for each $w(x) \in W_{2}^{1}[0,1]$. The approximate solution $v_{n}(x)$ is given as in (2.7) by taking finitely many terms. In other words, $v_{n}(x)$ is $n$-truncation of the Fourier series corresponding to $v(x)$.

\section{A Numerical Example}

We consider an illustrative example to demonstrate the accuracy of the proposed method and to test its utility. All computations are performed by using the Mathematica 7.0 software package.

Example 1. We investigate the approximate solution of the following nonlocal BVP:

$$
\begin{aligned}
& u^{(4)}(x)+e^{2 x} u^{\prime \prime \prime}(x)+e^{-x} u^{\prime \prime}(x)-e^{-3 x} u^{\prime}(x)+e^{x} u(x) \\
& \quad=\left(1+e^{-x}+e^{x}\right) \cosh (x)+\left(e^{2 x}-e^{-3 x}\right) \sinh (x), \quad 0 \leq x \leq 1,
\end{aligned}
$$




$$
\begin{aligned}
u\left(\frac{1}{5}\right) & +2 u^{\prime}\left(\frac{2}{5}\right)-u^{\prime \prime}\left(\frac{3}{5}\right)+3 u^{\prime \prime \prime}\left(\frac{4}{5}\right) \\
& +\int_{0}^{1}\left(u(s)+s^{2} u^{\prime}(s)-s u^{\prime \prime}(s)+e^{s} u^{\prime \prime \prime}(s)\right) d s \\
= & \cosh \left(\frac{1}{5}\right)-\cosh \left(\frac{3}{5}\right)+2 \sinh \left(\frac{2}{5}\right)+3 \sinh \left(\frac{4}{5}\right)-\frac{15}{4}+\frac{3}{e}+e+\frac{e^{2}}{4}, \quad(4.2) \\
-2 u & \left(\frac{1}{5}\right)-3 u^{\prime}\left(\frac{2}{5}\right)+u^{\prime \prime}\left(\frac{3}{5}\right)+u^{\prime \prime \prime}\left(\frac{4}{5}\right)+\int_{0}^{1}\left(s^{3} u(s)+s e^{s} u^{\prime}(s)-s u^{\prime \prime \prime}(s)\right) d s \\
= & -2 \cosh \left(\frac{1}{5}\right)+\cosh \left(\frac{3}{5}\right)-3 \sinh \left(\frac{2}{5}\right)+\sinh \left(\frac{4}{5}\right) \\
& +\frac{1}{8}\left(47-\frac{72}{e}-8 e+e^{2}\right) \\
4 u( & \left.\frac{1}{5}\right)-u^{\prime \prime}\left(\frac{3}{5}\right)+2 u^{\prime \prime \prime}\left(\frac{4}{5}\right)+\int_{0}^{1}\left(2 s u(s)+u^{\prime}(s)-s^{3} u^{\prime \prime}(s)+s u^{\prime \prime \prime}(s)\right) d s \\
= & 4 \cosh \left(\frac{1}{5}\right)-\cosh \left(\frac{3}{5}\right)+2 \sinh \left(\frac{4}{5}\right)-5+\frac{7}{e}+e+\cosh (1) \\
-3 & \left(\frac{2}{5}\right)+u^{\prime \prime}\left(\frac{3}{5}\right)-u^{\prime \prime \prime}\left(\frac{4}{5}\right) \\
& +\int_{0}^{1}\left((s-2) u(s)+(s-1)^{2} u^{\prime}(s)+u^{\prime \prime}(s)-u^{\prime \prime \prime}(s)\right) d s \\
= & \cosh \left(\frac{3}{5}\right)-3 \sinh \left(\frac{2}{5}\right)-\sinh \left(\frac{4}{5}\right)-1
\end{aligned}
$$

The exact solution to the problem (4.1)-(4.5) is determined by $u(x)=$ $\cosh (x)$. In our computations, $x_{i}=\frac{i-1}{n-1}(i=1,2, \ldots, n)$ and $n=11,21,31$. The numerical results computed via the presented method are compared with the exact solution of the example in Table 1 .

Table 1. The numerical results for Example 1.

\begin{tabular}{llllll}
\hline$x$ & $u(x)$ & $u_{31}(x)$ & $\left|u(x)-u_{11}(x)\right|$ & $\left|u(x)-u_{21}(x)\right|$ & $\left|u(x)-u_{31}(x)\right|$ \\
\hline 0. & 1. & 0.999965 & 0.000317719 & 0.0000788556 & 0.0000349928 \\
0.1 & 1.005 & 1.00496 & 0.000363112 & 0.0000898037 & 0.0000398235 \\
0.2 & 1.02007 & 1.02002 & 0.000425385 & 0.000104835 & 0.000046457 \\
0.3 & 1.04534 & 1.04528 & 0.000507836 & 0.000124769 & 0.0000552571 \\
0.4 & 1.08107 & 1.08101 & 0.00061306 & 0.000150252 & 0.0000665104 \\
0.5 & 1.12763 & 1.12755 & 0.000742959 & 0.000181756 & 0.0000804267 \\
0.6 & 1.18547 & 1.18537 & 0.000898774 & 0.00021959 & 0.0000971431 \\
0.7 & 1.25517 & 1.25505 & 0.00108114 & 0.000263914 & 0.000116731 \\
0.8 & 1.33743 & 1.3373 & 0.00129018 & 0.000314757 & 0.000139203 \\
0.9 & 1.43309 & 1.43292 & 0.00152555 & 0.000372039 & 0.000164523 \\
1. & 1.54308 & 1.54289 & 0.00178654 & 0.000435583 & 0.000192615 \\
\hline
\end{tabular}

Furthermore, the graphs of the absolute error functions for several approximations $u_{n}(x)$ are displayed in Figure 1. 


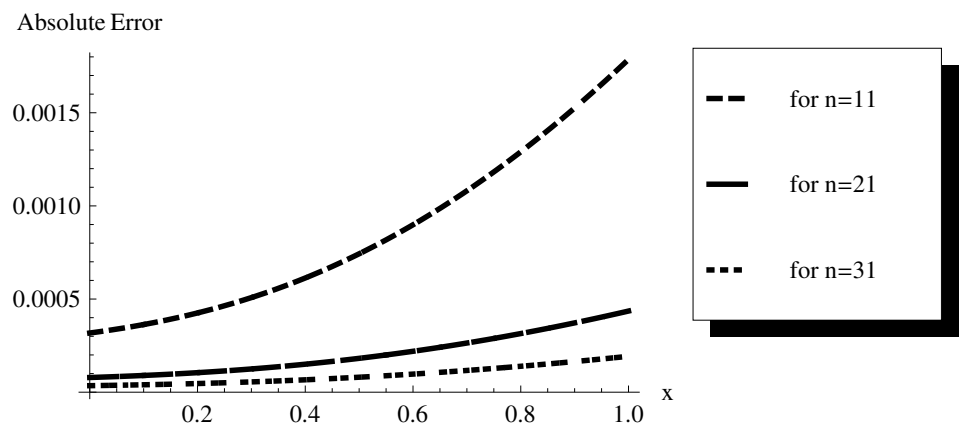

Figure 1. The graphs of the absolute error functions of the approximations $u_{n}(x)$ for Example 1 .

\section{Conclusions}

As can be seen from the values of absolute errors in Table 1 and the graphs in Figure 1, the numerical results verify that the algorithm used is highly accurate for solving fourth-order multi-point boundary value problems modeled by the linear ordinary differential equation involving nonlocal boundary conditions.

The method is still quite accurate and effective for the cases with both derivative and integral boundary conditions even if its accuracy is less compared to the cases with only derivative boundary conditions.

Clearly, the method has crucial advantages on determining the approximate solutions to these problems modeled by the equations with principally variable coefficients in considered form in this work and it can be used reliably. Therefore, it might be useful to the researchers requiring the approximate solutions of these model problems.

\section{Acknowledgements}

The authors are thankful to the anonymous reviewers for their valuable comments which contribute to improve the present paper.

\section{References}

[1] M.G. Cui and F.Z. Geng. Solving singular two-point boundary value problem in reproducing kernel space. J. Comput. Appl. Math., 205:6-15, 2007. http://dx.doi.org/10.1016/j.cam.2006.04.037.

[2] M.G. Cui and Y.Z. Lin. Nonlinear Numerical Analysis in Reproducing Kernel Space. Nova Science Publishers Inc., 2009.

[3] F.Z. Geng. A new reproducing kernel Hilbert space method for solving nonlinear fourth-order boundary value problems. Appl. Math. Comput., 213:163-169, 2009. http://dx.doi.org/10.1016/j.amc.2009.02.053.

[4] F.Z. Geng. Solving singular second order three-point boundary value problems using reproducing kernel Hilbert space method. Appl. Math. Comput., 215:20952102, 2009. http://dx.doi.org/10.1016/j.amc.2009.08.002. 
[5] F.Z. Geng and M.G. Cui. Solving a nonlinear system of second order boundary value problems. J. Math. Anal. Appl., 327:1167-1181, 2007. http://dx.doi.org/10.1016/j.jmaa.2006.05.011.

[6] F.Z. Geng and M.G. Cui. Solving singular nonlinear second-order periodic boundary value problems in the reproducing kernel space. Appl. Math. Comput., 192:389-398, 2007. http://dx.doi.org/10.1016/j.amc.2007.03.016.

[7] F.Z. Geng and M.G. Cui. Multi-point boundary value problem for optimal bridge design. Int. J. Comput. Math., 87(5):1051-1056, 2010.

http://dx.doi.org/10.1080/00207160903023573.

[8] C.L. Li and M.G. Cui. The exact solution for solving a class nonlinear operator equations in the reproducing kernel space. Appl. Math. Comput., 143(2-3):393399, 2003. http://dx.doi.org/10.1016/S0096-3003(02)00370-3.

[9] Y.Z. Lin and J.N. Lin. Numerical algorithm about a class of linear nonlocal boundary value problems. Appl. Math. Lett., 23:997-1002, 2010. http://dx.doi.org/10.1016/j.aml.2010.04.025.

[10] K. Orucoglu. A new Green's function concept for fourth-order differential equations. Electron. J. Differential Equations, 28:1-12, 2005.

[11] S. Roman. Linear differential equation with additional conditions and formulae for Green's function. Math. Model. Anal., 16(3):401-417, 2011. http://dx.doi.org/10.3846/13926292.2011.602125.

[12] S. Roman and A. Štikonas. Third-order linear differential equation with three additional conditions and formula for Green's function. Lithuanian Math. J., 50(4):426-446, 2010. http://dx.doi.org/10.1007/s10986-010-9097-x.

[13] I. Stakgold. Green's Functions and Boundary Value Problems. Wiley-Interscience Publications, New York, 1998.

[14] S. Timoshenko. Theory of Elastic Stability. McGraw-Hill, New York, 1961.

[15] A. Stikonas and S. Roman. Stationary problems with two additional conditions and formulae for Green's function. Numer. Funct. Anal. Optim., 30(9-10):11251144, 2009. http://dx.doi.org/10.1080/01630560903420932.

[16] B.Y. Wu and X.Y. Li. A new algorithm for a class of linear nonlocal boundary value problems based on the reproducing kernel method. Appl. Math. Lett., 24:156-159, 2011. http://dx.doi.org/10.1016/j.aml.2010.08.036.

[17] Y.F. Zhou, M.G. Cui and Y.Z. Lin. A computational method for nonlinear $2 m$ th order boundary value problems. Math. Model. Anal., 15(4):571-586, 2010. http://dx.doi.org/10.3846/1392-6292.2010.15.571-586. 